\title{
Three-Dimensional Memristive Hindmarsh-Rose Neuron Model with Hidden Coexisting Asymmetric Behaviors
}

\author{
Bocheng Bao $(\mathbb{D}$, Aihuang Hu, Han Bao, Quan Xu, Mo Chen, and Huagan Wu \\ School of Information Science and Engineering, Changzhou University, Changzhou 213164, China \\ Correspondence should be addressed to Bocheng Bao; mervinbao@126.com
}

Received 27 December 2017; Accepted 24 January 2018; Published 28 February 2018

Academic Editor: Viet-Thanh Pham

Copyright (C) 2018 Bocheng Bao et al. This is an open access article distributed under the Creative Commons Attribution License, which permits unrestricted use, distribution, and reproduction in any medium, provided the original work is properly cited.

\begin{abstract}
Since the electrical activities of neurons are closely related to complex electrophysiological environment in neuronal system, a novel three-dimensional memristive Hindmarsh-Rose (HR) neuron model is presented in this paper to describe complex dynamics of neuronal activities with electromagnetic induction. The proposed memristive HR neuron model has no equilibrium point but can show hidden dynamical behaviors of coexisting asymmetric attractors, which has not been reported in the previous references for the HR neuron model. Mathematical model based numerical simulations for hidden coexisting asymmetric attractors are performed by bifurcation analyses, phase portraits, attraction basins, and dynamical maps, which just demonstrate the occurrence of complex dynamical behaviors of electrical activities in neuron with electromagnetic induction. Additionally, circuit breadboard based experimental results well confirm the numerical simulations.
\end{abstract}

\section{Introduction}

In the past three decades, numerous simplified neuron models had been fantastically extended from the classical HodgkinHuxley model [1] to reconstruct the main dynamical characteristics of neuronal electrical activities [2-8], among which the two- and three-dimensional Hindmarsh-Rose (HR) neuron models are effective and available for dynamical analysis in electrical activities of biological neurons $[9,10]$. In the last few years, a wide variety of the HR neuron models, such as original three-dimensional HR models [1016], extended or nonlinear feedback coupled HR models [1720], time delayed HR models [20-22], fractional-order HR models $[23,24]$, and memristor based HR models under electromagnetic radiations $[9,25-27]$, have been proposed and further studied by bifurcation analysis methods for understanding the dynamics of electrical activities among neurons [8]. For this reason, bifurcation analysis theory plays an essential role in describing mode transitions between spiking and bursting in the neuronal electrical activities [927].

Inspired by the constructing approach of the threedimensional HR neuron model $[3,24]$, a novel threedimensional memristive HR neuron model is presented in this paper, which could be used to better describe complex dynamical characteristics of neuronal electrical activities with electromagnetic induction or further exhibit some undiscovered complex dynamical behaviors in neuronal electrical activities. Interestingly, our proposed memristive HR neuron model has no equilibrium point, which can be classified as a particular dynamical system with hidden oscillating patterns [28-31]. Furthermore, coexisting asymmetric attractors' behavior can also be observed in such memristive HR neuron model as well, indicating the emergence of bistability dynamics, which has been found in some specified neuron models [32-35]. However, the phenomenon of hidden coexisting asymmetric attractors has not been previously reported for the HR neuron model.

This paper is organized as follows. In Section 2, based on the brief reviews on the HR neuron model, a threedimensional memristive HR neuron model is presented, upon which hidden coexisting asymmetric attractors are numerically revealed by phase portraits and time series and its bistability dynamics are confirmed by the attraction basins related to the initial values. In Section 3, hidden coexisting asymmetric attractors' behaviors are demonstrated by bifurcation diagrams, Lyapunov exponents, and dynamical maps, from which numerous types of coexisting asymmetric 
attractors are easily observed. In addition, a physical implementation circuit is fabricated and breadboard experiments are carried out to confirm the hidden coexisting asymmetric attractors in Section 4. The conclusions are summarized in Section 5.

\section{Three-Dimensional Memristive HR Neuron Model}

2.1. Brief Reviews on the HR Neuron Model. Through simplifying the classical Hodgkin-Huxley model [1], the twodimensional Hindmarsh-Rose (HR) neuron model was proposed by Hindmarsh and Rose [2] in 1982, which is described by two first-order ordinary differential equations as

$$
\begin{aligned}
& \dot{x}=y-a x^{3}+b x^{2}+I \\
& \dot{y}=c-d x^{2}-y,
\end{aligned}
$$

in which two variables $x$ and $y$ are the membrane potential and recovery variable (also called spiking variable), respectively, and a term $I$ is the externally applied current. The parameters $a, b, c$, and $d$ are four positive constants, which are often assumed as $a=1, b=3, c=1$, and $d=5$, respectively $[2,11-14]$.

To permit numerous dynamical behaviors, for example, chaotic dynamics, for the membrane potential, an extra third equation was introduced by Hindmarsh and Rose [3] in 1984 to improve the two-dimensional neuron model (1), which is expressed by three first-order ordinary differential equations as

$$
\begin{aligned}
& \dot{x}=y-a x^{3}+b x^{2}+I-z \\
& \dot{y}=c-d x^{2}-y \\
& \dot{z}=r\left(s\left(x-x_{1}\right)-z\right),
\end{aligned}
$$

where the variable $z$ is the bursting variable and the constant $x_{1}$ is the resting potential of the model. The newly added parameters $r$ and $s$ are two positive constants but $r$ is very small. Thus, a new variable $z$, a slowly evolving current, is coupled into the first equation of the two-dimensional model (1) to tune the externally applied current $I$. If the threedimensional neuron model (2) is in its firing state, the value of $z$ increases [24].

\subsection{Constructed Memristive HR Neuron Model. Motivated by} the above constructing approach of the model (2), a threedimensional memristive HR neuron model with electromagnetic induction effect is proposed through introducing a flux-controlled ideal memristor into the first equation of the model (1), which can be mathematically modeled by

$$
\begin{aligned}
& \dot{x}=y-a x^{3}+b x^{2}+I+k \varphi x \\
& \dot{y}=c-d x^{2}-y \\
& \dot{\varphi}=x,
\end{aligned}
$$

where the new variable $\varphi$ is the magnetic flux indicating the time integral of the membrane potential $x$. The newly adding term $k \varphi x$ represents the externally applied electromagnetic induction and $k$ is the strength of the electromagnetic induction.

It is important to stress that the introduced memristor in (3) is ideal and flux-controlled. According to the definitions of ideal memristor by state-dependent Ohm's laws between the terminal voltage $v$ and terminal current $i[36,37]$, a fluxcontrolled ideal memristor $W$ is thereby given as

$$
\begin{aligned}
i & =W(\varphi) v, \\
\dot{\varphi} & =v,
\end{aligned}
$$

where the memductance $W(\varphi)$ can be interpreted as the flux-dependent rate of change of charge. Therefore, the memductance $W(\varphi)$ utilized in (3) can be written by

$$
W(\varphi)=k \varphi
$$

in which the coefficient $k$ is positive.

To exhibit three characteristic fingerprints of pinched hysteresis loop of the memristor modeled by (4) and (5) [38], a sinusoidal voltage source $v=A \sin (2 \pi F \tau)$ is connected at the input terminals of the memristor, where $A$ and $F$ are the amplitude and frequency, respectively. Let $k=1$. When $A=4$ is maintained unchanged and $F$ is assigned as $0.1,0.2$, and 0.5 , respectively, the $v-i$ plots are shown in Figure 1(a), while when $F=0.1$ is fixed and $A$ is determined as 3,4 , and 5 , respectively, the $v-i$ plots are shown in Figure 1(b). It is seen from Figure 1 that the $v-i$ plots are the hysteresis loops pinched at the origin. The hysteresis loop is pinched regardless of the stimulus amplitudes but shrinks into a linear function at infinite frequency and its lobe area decreases with increasing the frequency. The numerical results in Figure 1 indicate that the memristor modeled by (4) can behave three fingerprints for distinguishing memristors [38].

In the next work, the three-dimensional memristive HR neuron model given in (3) is considered. It should be remarked that the adjustable parameters of interest are $I$ and $k$, and their regions are correspondent to the first quadrant of the parameter space $(I>0$ and $k>0)$. For any uncertain parameter $I$, the existence of any equilibrium point is not allowed in the three-dimensional memristive HR neuron model, neither stable nor unstable. Only if the applied current $I=-1$ will the model show an equilibrium point, which is not in the considered parameter region. This case is often encountered in various kinds of nonlinear dynamical systems that are known to generate the specified hidden attractors [28-31].

2.3. Coexisting Asymmetric Attractors. When the original parameters are selected as $a=1, b=3, c=1$, and $d=5$, respectively [2], an example for model (3) with $I=1$ and $k=0.9$ is given as shown in Figure 2, where the orbits marked by the red and blue colors emerge from the initial values $(0,0,-2)$ and $(0,0,2)$, respectively. In Figure 2(a), the phase portraits in the $x-\varphi$ plane display the bistability phenomenon of hidden coexisting asymmetric 


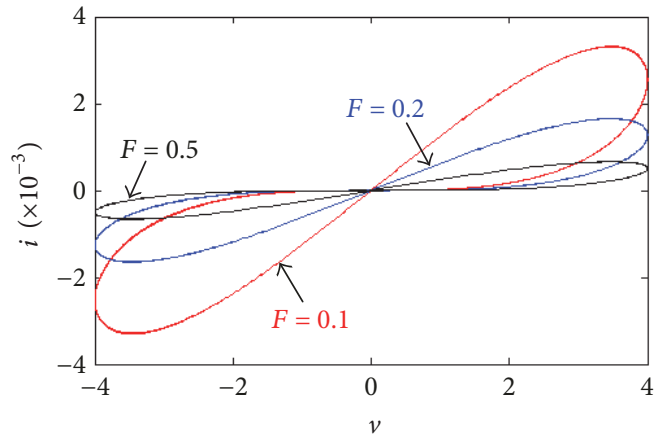

(a)

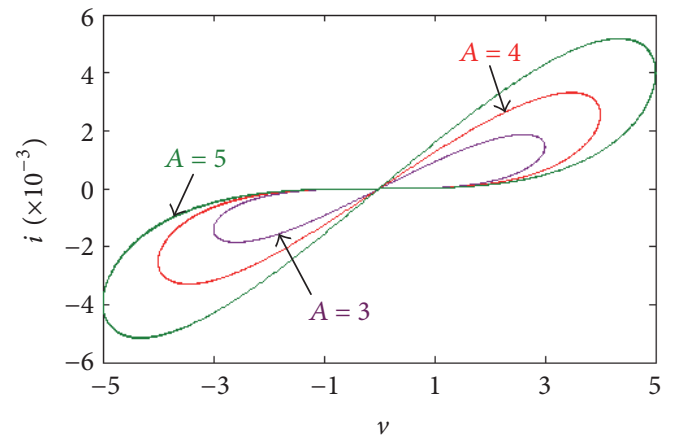

(b)

Figure 1: Pinched hysteresis loops of the flux-controlled ideal memristor. (a) $A=4$ with $F=0.1,0.2$, and 0.5 . (b) $F=0.1$ with $A=3,4$, and 5.

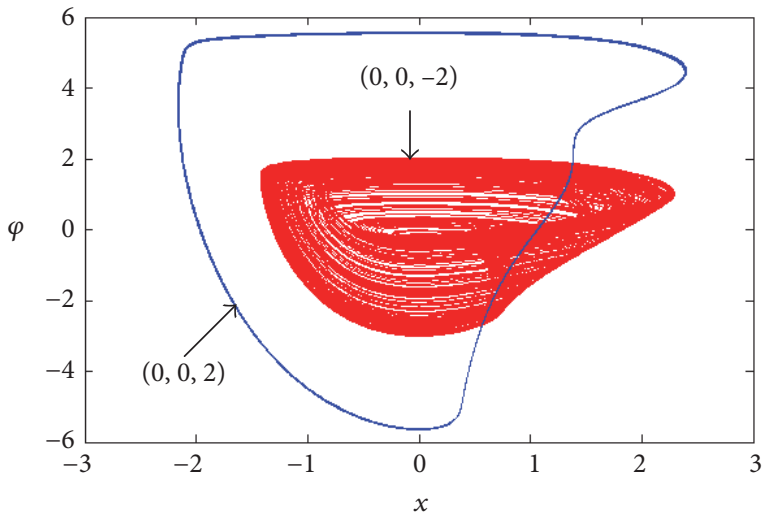

(a)
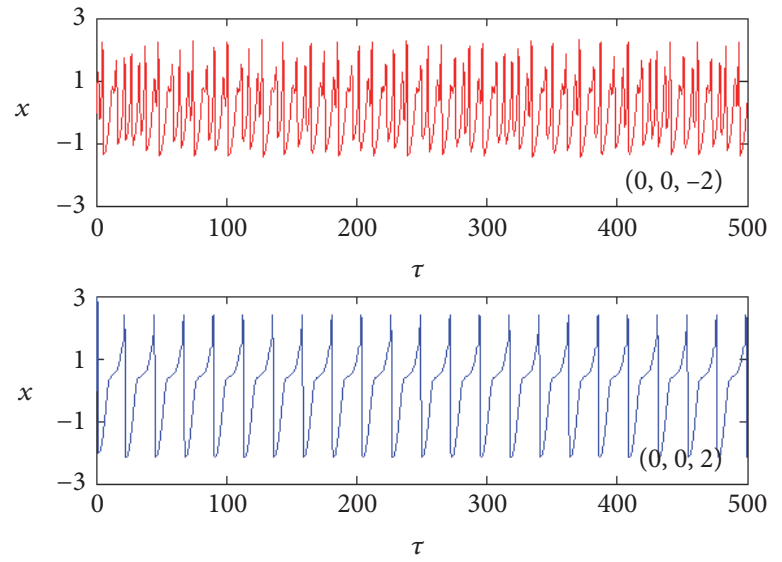

(b)

Figure 2: Hidden coexisting asymmetric attractors emerged from the initial values $(0,0,-2)$ and $(0,0,2)$. (a) Phase portraits in the $x$ - $\varphi$ plane. (b) Time series of the variable $x$.

attractors consisting of chaotic attractor and limit cycle in the memristive HR neuron model, whereas in Figure 2(b), the time series of the membrane potential $x$ demonstrate the coexistence of chaotic and periodic spikes in the memristive HR neuron model as well. Correspondingly, three Lyapunov exponents for the initial values $(0,0,-2)$ are $0.0782,0$, and -3.0684 , respectively, while those for $(0,0,2)$ are 0 , -0.2717 , and -2.8556 , respectively. Remark that Wolf et al.s method [39] with MATLAB ODE113 algorithm is here used to calculate three Lyapunov exponents.

For the coexisting asymmetric attractors shown in Figure 2(a), the corresponding attraction basins in the $x(0)-\varphi(0)$ and $x(0)-y(0)$ planes of the initial values are drawn in Figures 3(a) and 3(b), where the attraction basins for chaotic attractors and periodic limit cycles are colored in the fuchsia and cyan regions, respectively. The results effectively indicate the emergence of bistability phenomenon in the memristive HR neuron model.

Particularly, the emerging coexisting asymmetric attractors do not associate with any equilibrium point, indicating that the memristive HR neuron model always operates in hidden oscillating patterns [28-31]. Additionally, it is interesting to note that, just like the self-excited coexisting asymmetric attractors in hyperbolic-type memristor based Hopfield neural network [32], such hidden coexisting asymmetric attractors in the memristive HR neuron model are induced by electromagnetic induction also, which illustrates the occurrence of complex dynamical behaviors of electrical activities in neuron with electromagnetic induction.

\section{Hidden Coexisting Asymmetric Attractors' Behavior}

When the applied current $I$ and electromagnetic induction strength $k$ are considered as two bifurcation parameters, hidden coexisting asymmetric behaviors of the memristive HR neuron model are numerically studied by MATLAB ODE45 algorithm under two sets of the initial values $(0,0$, $-2)$ and $(0,0,2)$.

3.1. Bifurcation Behaviors with Increasing $k$. Figure 4 gives the bifurcation diagrams of $x$ and the first two Lyapunov exponents as $I=1$ and $k=0.5 \sim 1.4$, where in Figure 4(a) the orbits marked by the red and blue colors emerge from 


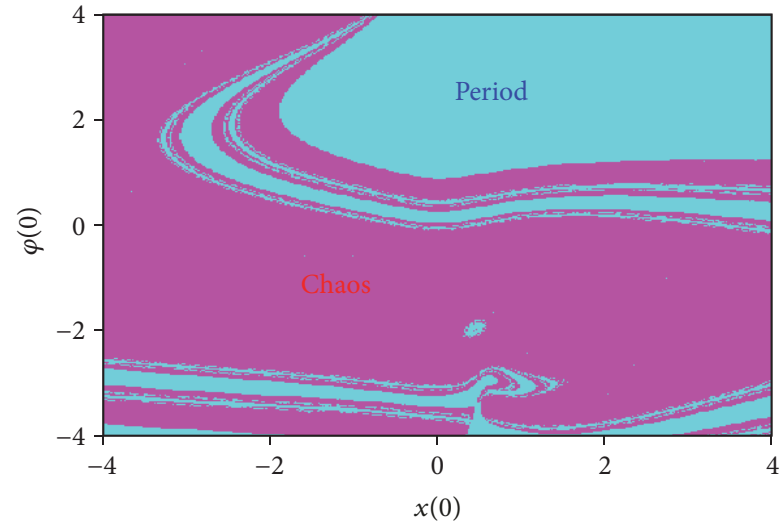

(a)

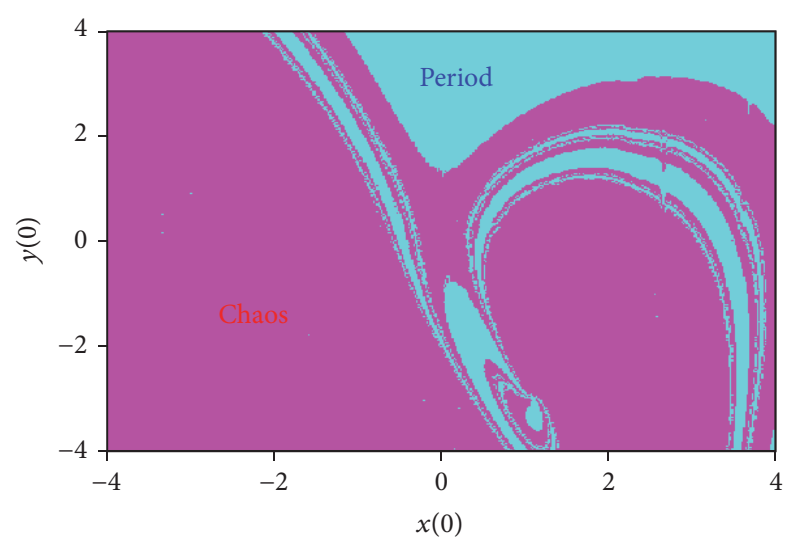

(b)

FIGURE 3: Attraction basins in two different planes for $I=1$ and $k=0.9$, indicating the emergence of bistability phenomenon. (a) The $x(0)-\varphi(0)$ plane with $y(0)=0$. (b) The $x(0)-y(0)$ plane with $\varphi(0)=0$.

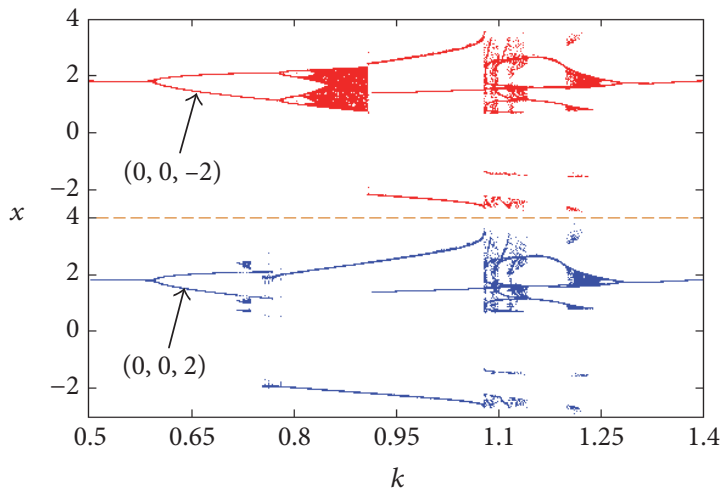

(a)

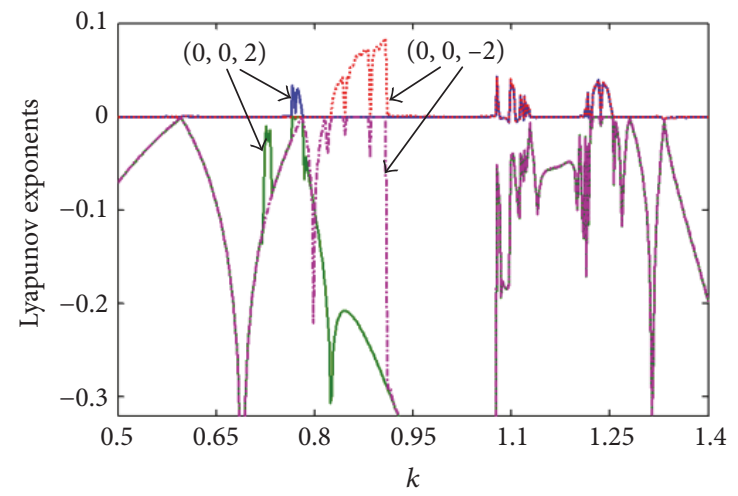

(b)

Figure 4: For the initial values $(0,0,-2)$ and $(0,0,2)$, hidden coexisting asymmetric behaviors with $I=1$ and $k=0.5 \sim 1.4$. (a) Bifurcation diagrams of $x$. (b) First two Lyapunov exponents.

the initial values $(0,0,-2)$ and $(0,0,2)$, respectively, and in Figure 4(b) the Lyapunov exponents marked by the red and fuchsia colors correspond to the initial values $(0,0,-2)$ and those marked by blue and green colors correspond to $(0,0,2)$. It can be seen from Figure 4 that complex dynamics appear in the memristive HR neuron model, in which chaotic attractors with different topologies, limit cycles with different periodicities, period-doubling bifurcation routes, tangent bifurcation routes, crisis scenarios, coexisting bifurcation modes, and so on can be found. Therefore, the electromagnetic induction by the introduced memristor induces numerous complex dynamics for the membrane potential $x$, especially including hidden coexisting asymmetric behaviors.

The concernedly coexisting behaviors of asymmetric attractors mainly locate in two parameter regions $[0.718,0.736]$ and $[0.754,0.909]$, in which some different types of hidden coexisting asymmetric attractors occur. When $I=1$ and $k=0.735$ and 0.81 , respectively, the phase portraits in the $x-\varphi$ plane for the other two types of hidden coexisting asymmetric attractors are depicted in Figure 5. In detail, Figure 5(a) exhibits the coexistence of hidden chaotic attractor and hidden limit cycle, and Figure 5(b) displays the coexistence of two hidden limit cycles with different periodicities.

3.2. Bifurcation Behaviors with Increasing I. Figure 6 demonstrates the bifurcation diagrams of $x$ and the first two Lyapunov exponents as $I=0 \sim 2.4$ and $k=0.9$, where the initial values for different colored orbits in Figure 6(a) and different colored Lyapunov exponents in Figure 6(b) are consistent with those used in Figures 4(a) and 4(b). In the same way, it can be observed from Figure 6 that complex dynamics are coined in the memristive HR neuron model, reflecting the dynamical effect of the externally applied current $I$ in neurons.

The parameter region $[0.98,1.65]$ has the benefit for the coexisting behaviors of asymmetric attractors, in which several different types of hidden coexisting asymmetric attractors can be clearly found. When $k=0.9$ is fixed and $I$ is set to 1.15 and to 1.62 , respectively, the phase portraits in the $x-\varphi$ plane for the two types of hidden coexisting asymmetric attractors are plotted in Figure 7, where in Figure 7(a) the 


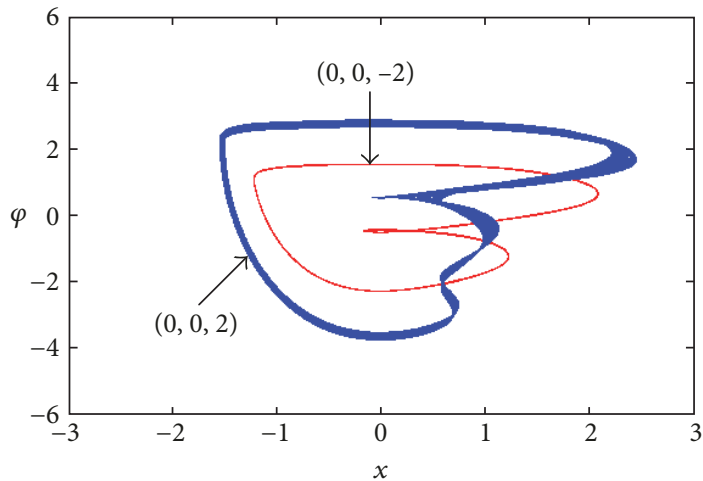

(a)

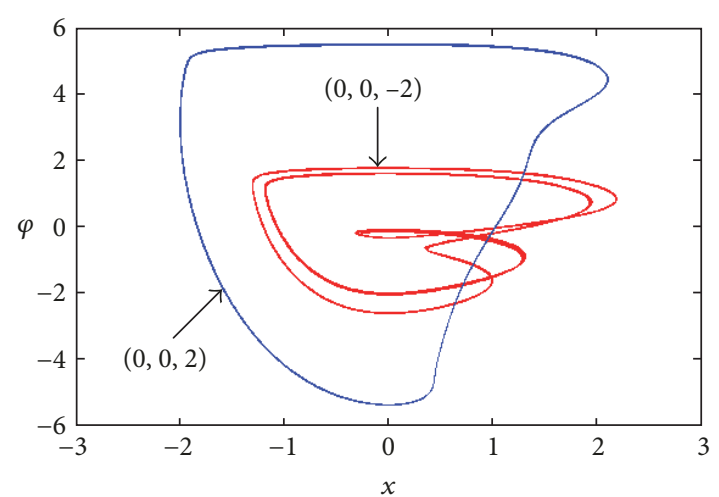

(b)

FIGURE 5: Phase portraits in the $x-\varphi$ plane for the other two types of hidden coexisting asymmetric attractors. (a) $I=1$ and $k=0.735$. (b) $I=$ 1 and $k=0.81$.

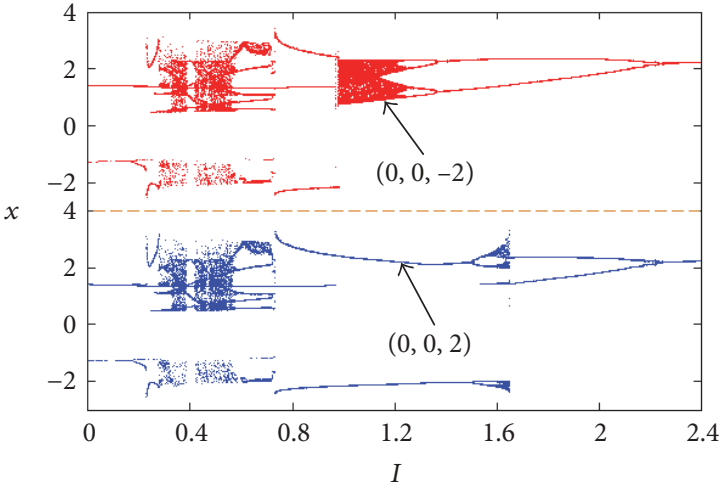

(a)

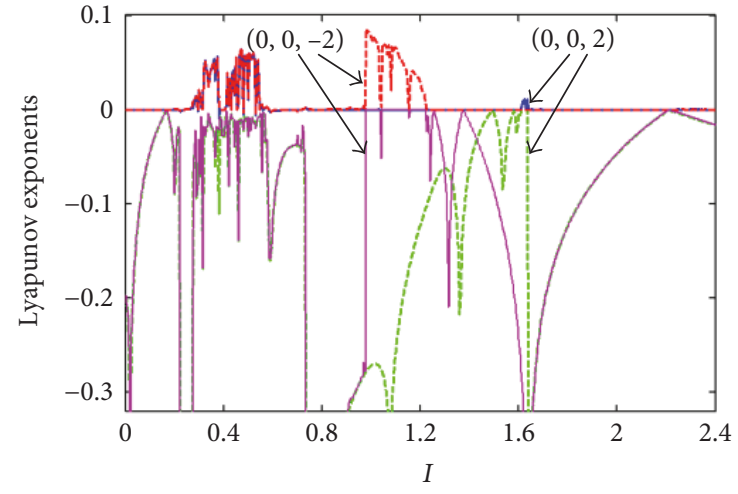

(b)

Figure 6: For the initial values $(0,0,-2)$ and $(0,0,2)$, hidden coexisting asymmetric behaviors with $I=0 \sim 2.4$ and $k=0.9$. (a) Bifurcation diagrams of $x$. (b) First two Lyapunov exponents.

coexistence of hidden chaotic attractor and hidden period 1 limit cycle is displayed, and in Figure 7(b) the coexistence of hidden period 2 limit cycle and hidden chaotic attractor with large size is presented.

3.3. Coexisting Asymmetric Behaviors in the Parameter Space. For intuitively manifesting the coexisting behaviors of asymmetric attractors in the memristive HR neuron model, dynamical maps depicted by the largest Lyapunov exponent under two sets of the initial values are numerically plotted in the $k-I$ parameter space [40], as shown in Figures 8(a) and $8(\mathrm{~b})$, where the luminous yellow, red, and black colored regions stand for the chaotic, periodic, and divergent behaviors, respectively. Figure 8 indicates how dynamical evolution in the electromagnetic induction strength $k$ and applied current $I$ affects the coexisting behaviors under different initial values being considered. When the two parameters $k$ and $I$ are evolved, some chaotic regions are embedded in the periodic regions; however, different chaotic regions appear on the parameter spaces of Figures 8(a) and 8(b), which are caused by the coexisting asymmetric attractors' behaviors under different initial values. The numerical results in Figure 8 illustrate that the dynamical behaviors depicted by the largest Lyapunov exponent based dynamical maps are well agreed with those revealed by the bifurcation behaviors in Figures 4 and 6.

It should be remarkable for the dynamical maps in Figure 8 that the chaotic attractors in different locations of the chaotic region have different topologies and the limit cycles in different locations of the periodic region have different periodicities. Specifically, except for several types of coexisting asymmetric behaviors shown in Figures 2, 5, and 7 , another type of coexisting asymmetric behaviors of chaotic attractor and divergent orbit can also be uncovered, which means that another form of bistability exists in the memristive HR neuron model.

\section{Circuit Design and Breadboard Experiments}

4.1. Physical Circuit Designs and Parameter Selections. The flux-controlled ideal memristor $W$ characterized by (4) and (5) and its constructing three-dimensional memristive HR neuron model expressed by (3) can be physically realized by using an electronic circuit via analog multipliers and operational amplifiers connected with resistors and/or capacitors [41-43], as drawn in Figures 9(a) and 9(b), respectively. Of 


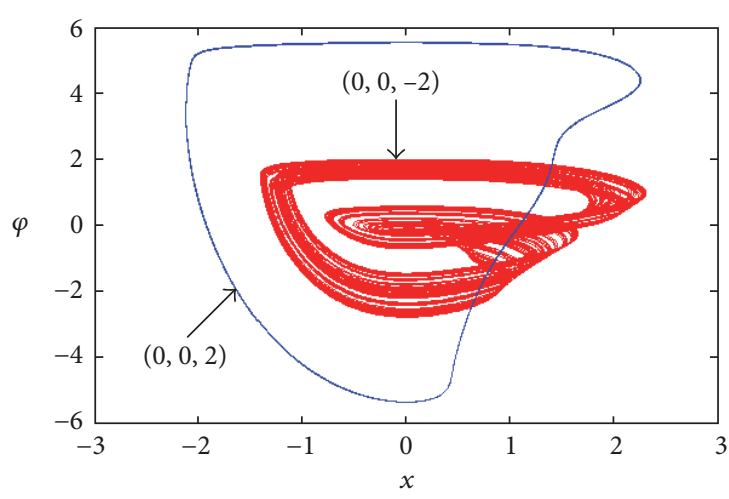

(a)

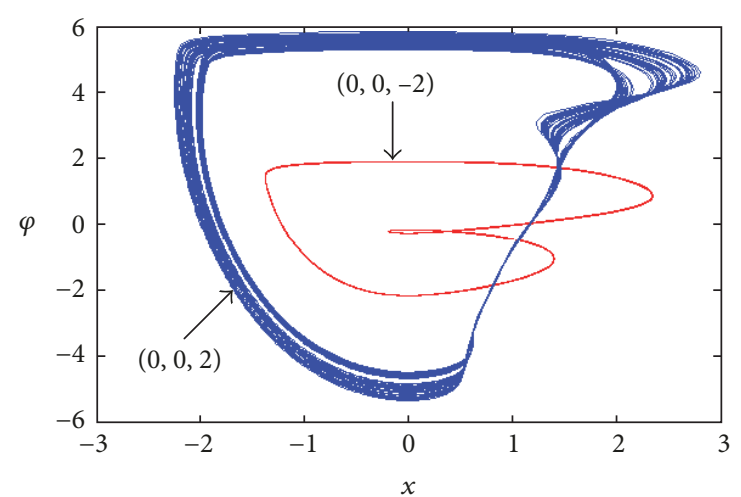

(b)

Figure 7: Phase portraits in the $x-\varphi$ plane for the other two types of hidden coexisting asymmetric attractors. (a) $I=1.15$ and $k=0.9$. (b) $I=$ 1.62 and $k=0.9$.

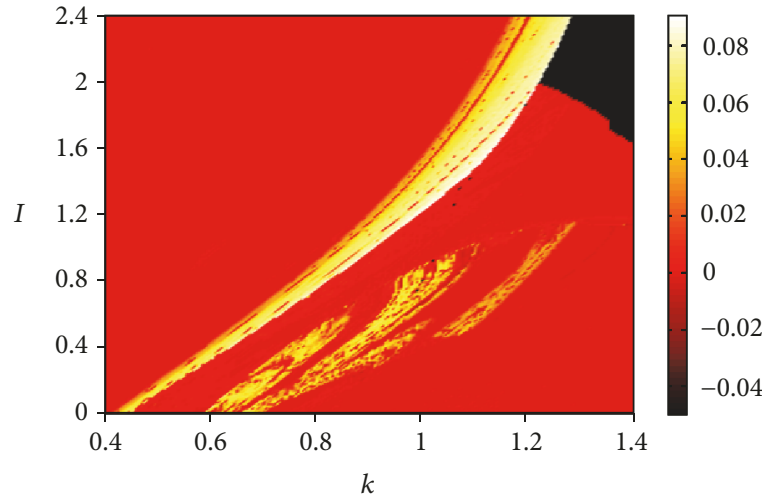

(a)

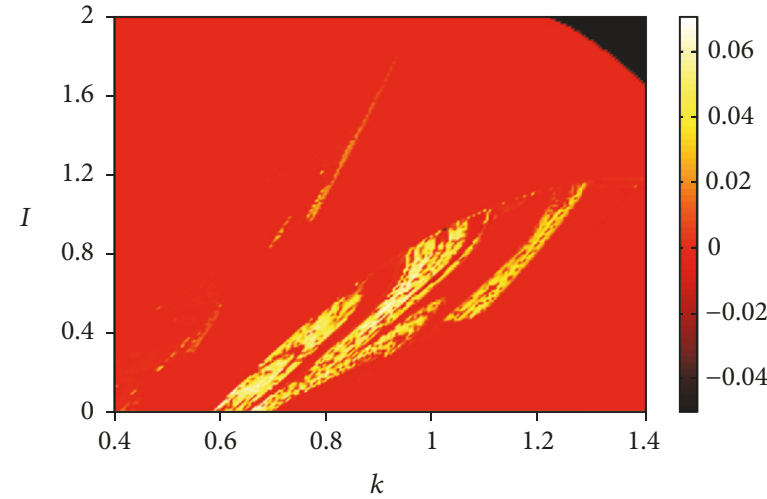

(b)

FIGURE 8: In the $k$-I parameter space, dynamical maps depicted by the largest Lyapunov exponent under different initial values. (a) Dynamical map for the initial values $(0,0,-2)$. (b) Dynamical map for the initial values $(0,0,2)$.

course, this three-dimensional memristive HR neuron model also can be digitally implemented in field-programmable gate arrays (FPGA) as well $[44,45]$.

The implementation circuit of the flux-controlled ideal memristor in Figure 9(a) contains an integrator with time constant $R C$, an inverter, a multiplier $M_{0}$, and a resistor $R_{k}$. For the input voltage $v$ and output current $i$, the mathematical model for the memristor emulator can be easily given as

$$
\begin{aligned}
i & =W\left(v_{\varphi}\right) v=\frac{g_{0}}{R_{k} C} v_{\varphi} v=\frac{1}{R C} \cdot k v_{\varphi} v \\
\dot{v}_{\varphi} & =\frac{1}{R C} \cdot v,
\end{aligned}
$$

where $v_{\varphi}$ is the inner variable of the memristor emulator and $g_{0}$ is the gain of the multipliers $M_{0}, k=g_{0} R / R_{k}$, and $W\left(v_{\varphi}\right)=$ $k v_{\varphi} / R C$.

The main circuit of the memristive HR neuron model in Figure 9(b) has two integrating channels for implementing the first and second equations of (3). According to Kirchhoff's circuit laws and electrical properties of the circuit components, the circuit equations of Figure 9(b) are written as

$$
\begin{aligned}
& R C \frac{\mathrm{d} v_{x}}{\mathrm{~d} t}= \frac{R}{R_{2}} v_{y}-\frac{g_{1} g_{2} R}{R_{4}} v_{x}^{3}+\frac{g_{2} R}{R_{3}} v_{x}^{2}+\frac{R}{R_{1}} V_{I} \\
&+\frac{g_{0} R}{R_{k}} v_{\varphi} v_{x} \\
& R C \frac{\mathrm{d} v_{y}}{\mathrm{~d} t}= \frac{R}{R_{6}} V_{y 0}-\frac{g_{3} R}{R_{5}} v_{x}^{2}-\frac{R}{R_{7}} v_{y} \\
& R C \frac{\mathrm{d} v_{\varphi}}{\mathrm{d} t}=v_{x},
\end{aligned}
$$

where $v_{x}$ and $v_{y}$ are two circuit variables, $V_{I}$ and $V_{y 0}$ are two applied voltages, and $g_{1}, g_{2}$, and $g_{3}$ are the gains of the multipliers $M_{1}, M_{2}$, and $M_{3}$, respectively.

Considering that the dynamic amplitude of the recovery variable $y$ in the numerical simulations exceeds the linear operation ranges of operational amplifier and multiplier, the following linear transformation

$$
\left(v_{x}, v_{y}, v_{\varphi}\right) \longrightarrow\left(v_{x}, 2.5 v_{y}, v_{\varphi}\right)
$$




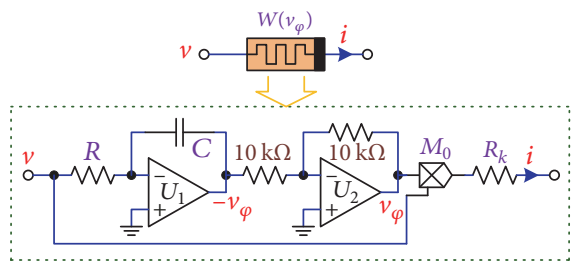

(a)

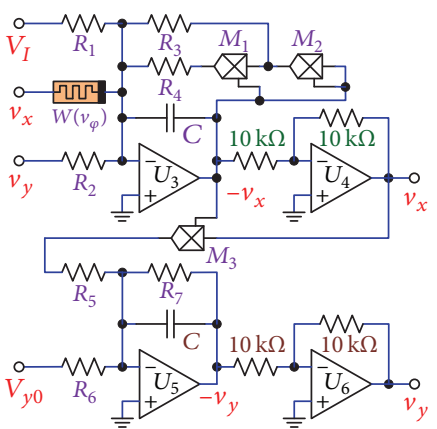

(b)

Figure 9: Physical electronic circuit implementation for the memristive HR neuron model. (a) Implementation circuit of the flux-controlled ideal memristor. (b) Main circuit of the memristive HR neuron model.

should be utilized to reduce the dynamic voltage amplitude of $v_{y}$ in the circuit equations of the memristive HR neuron model. Thus, by comparing (8) with (3), there yields

$$
\begin{aligned}
& R_{1}=\frac{R}{I}, \\
& R_{k}=\frac{g_{0} R}{k}, \\
& R_{2}=\frac{R}{2.5}, \\
& R_{3}=\frac{g_{2} R}{3}, \\
& R_{4}=g_{1} g_{2} R, \\
& R_{5}=\frac{g_{3} R}{2}, \\
& R_{6}=2.5 R, \\
& R_{7}=R .
\end{aligned}
$$

Let the time constant $R C=10 \mathrm{k} \Omega \times 33 \mathrm{nF}=330 \mu \mathrm{s}$; that is, $R=10 \mathrm{k} \Omega$ and $C=33 \mathrm{nF}$ and the multiplier gains $g_{0}=g_{1}=$ 0.1 and $g_{2}=g_{3}=1$. According to (9), the circuit parameters for the breadboard experiments of the memristive HR neuron model can be calculated, as listed in Table 1 .

4.2. Results Captured from Breadboard Experiments. According to the circuit diagrams in Figure 9 and circuit parameters in Table 1, a hardware circuit using commercially discrete components can be welded on a breadboard. The operational amplifiers AD711JN and analog multipliers AD633JN supplied by $\pm 15 \mathrm{~V}$ voltage modules are chosen. The DC voltages $V_{I}$ and $V_{y 0}$ are provided by Tektronix PWS $2326 \mathrm{DC}$ Power Supply and the experimental results are measured by Tektronix TDS 3054C Digital Phosphor Oscilloscope. The photograph of the connectedly experimental prototype for the memristive HR neuron model is displayed in Figure 10.

For experimentally measuring the pinched hysteresis loops of the memristor emulator given in Figure 9(a),
TABLE 1: Circuit parameters of the memristive HR neuron model for breadboard experiments.

\begin{tabular}{lcc}
\hline Parameters & Significations & Values \\
\hline$R, R_{7}$ & Resistance & $10 \mathrm{k} \Omega$ \\
$R_{1}$ & Resistance & $10 \mathrm{k} \Omega$ (adjustable) \\
$R_{k}$ & Resistance & $1.11 \mathrm{k} \Omega$ (adjustable) \\
$R_{2}$ & Resistance & $4 \mathrm{k} \Omega$ \\
$R_{3}$ & Resistance & $3.3 \mathrm{k} \Omega$ \\
$R_{4}$ & Resistance & $1 \mathrm{k} \Omega$ \\
$R_{5}$ & Resistance & $5 \mathrm{k} \Omega$ \\
$R_{6}$ & Resistance & $25 \mathrm{k} \Omega$ \\
$C$ & Capacitance & $33 \mathrm{nF}$ \\
$V_{I}, V_{y 0}$ & DC voltage & $1 \mathrm{~V}$ \\
$g_{0}, g_{1}$ & Multiplier gain & 0.1 \\
$g_{2}, g_{3}$ & Multiplier gain & 1 \\
\hline
\end{tabular}

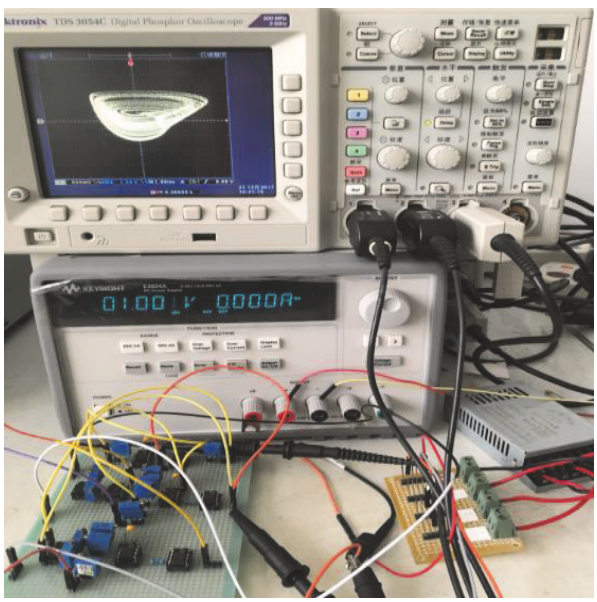

FIGURE 10: Photograph of the experimental breadboard and typical chaotic attractor captured by the digital oscilloscope.

a sinusoidal voltage source $v=A \sin (2 \pi f t)$ generated by Tektronix AFG 3102C Function Generator is linked to the input terminals of the memristor emulator, where the physical frequency is calculated by $f=F / R C$. For $k=1$, the adjustable 


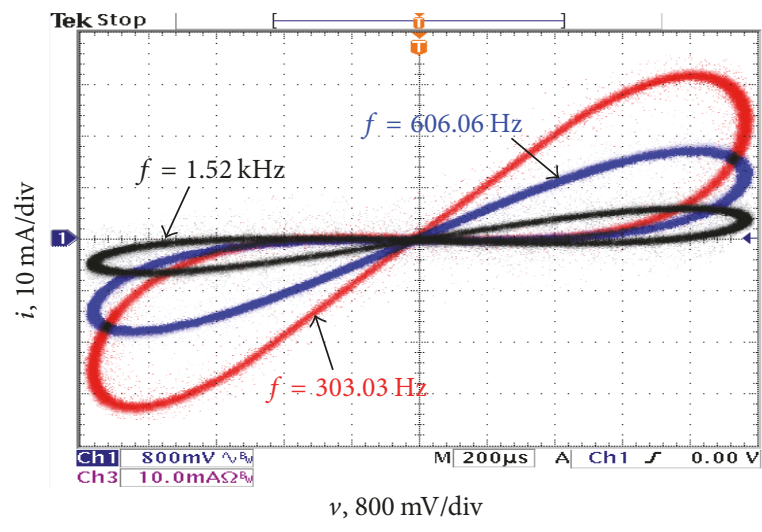

(a)

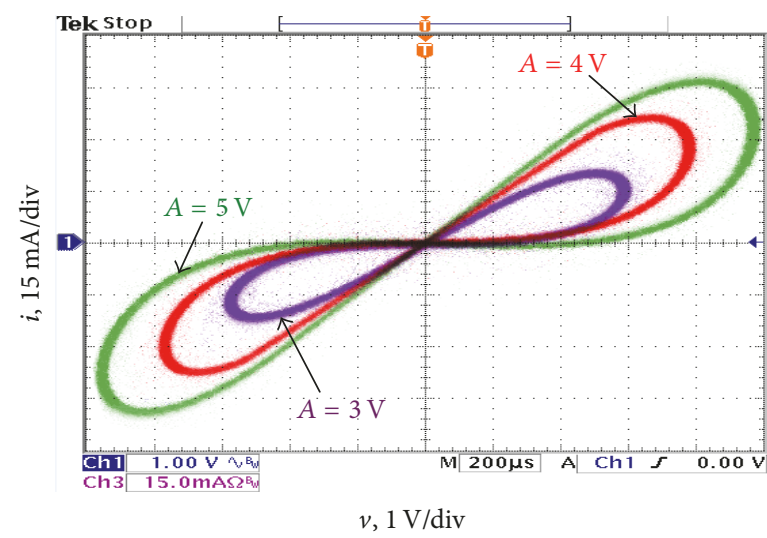

(b)

FIGURE 11: Experimentally measured pinched hysteresis loops of the memristor emulator. (a) $A=4 \mathrm{~V}$ with different frequencies. (b) $f=$ $303.03 \mathrm{~Hz}$ with different amplitudes.

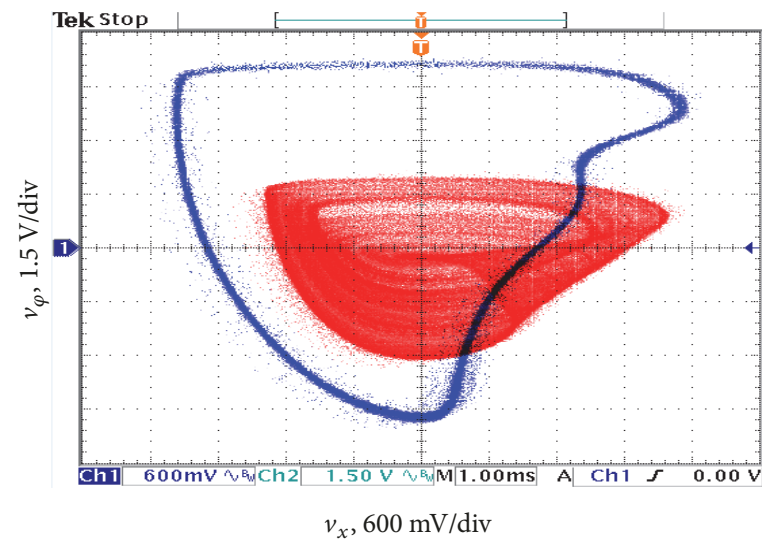

(a)

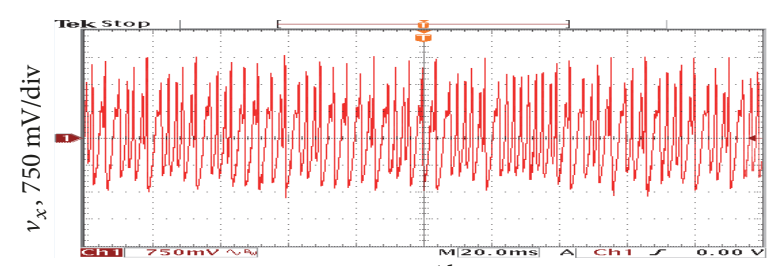

$t, 20 \mathrm{~ms} / \mathrm{div}$

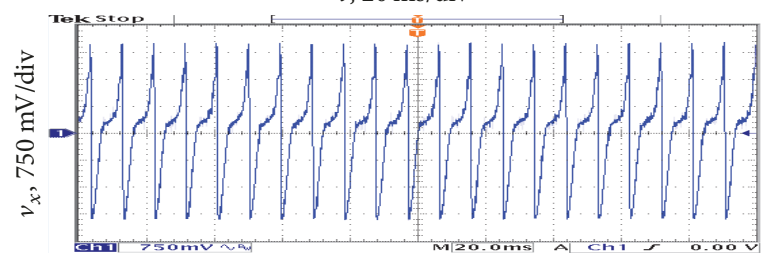

$t, 20 \mathrm{~ms} / \mathrm{div}$

(b)

FIGURE 12: Experimentally measured hidden coexisting asymmetric attractors while repeatedly switching on and off the experimental power supply. (a) Phase portraits in the $v_{x}-v_{\varphi}$ plane. (b) Time series of the variable $v_{x}$.

resistance $R_{k}=10 \mathrm{k} \Omega$. When the amplitudes and frequencies of the sinusoidal voltage source used during numerical simulations in Figure 1 are employed, the pinched hysteresis loops for the corresponding amplitudes and frequencies are captured, as shown in Figures 11(a) and 11(b), respectively, which experimentally validate the characteristic fingerprints of the memristor emulator. It should be addressed that for better observing the experimental results, all the output currents sensed by the current probe are magnified by ten times.

The circuit parameters listed in Table 1 are used and the different initial voltages of three capacitors are randomly sensed by repeatedly switching on and off the experimental power supply [46]. For the typical circuit parameters in Table 1, two adjustable circuit parameters of $R_{1}$ and $R_{k}$ correspond to the adjustable model parameters of $I=1$ and $k=0.9$. Corresponding to Figure 2, the phase portraits in the $v_{x}-v_{\varphi}$ plane and time series of the variable $v_{x}$ that emerged from different initial voltages are experimentally obtained, as shown in Figure 12. The experimental results indicate that hidden coexisting asymmetric attractors also can be measured from the breadboard experiments of the memristive HR neuron model as well.

When the applied current $I=1$, that is, the resistance $R_{1}$ is fixed as $10 \mathrm{k} \Omega$ and the resistance $R_{k}$ is set to $1.36 \mathrm{k} \Omega$ and to $1.23 \mathrm{k} \Omega$, respectively, the phase portraits in the $v_{x}-v_{\varphi}$ plane are captured, as shown in Figures 13(a) and 13(b). Furthermore, when the electromagnetic induction strength $k=0.9$, that is, $R_{k}=1.11 \mathrm{k} \Omega$ and $R_{1}$ is set to $8.70 \mathrm{k} \Omega$ and to $6.17 \mathrm{k} \Omega$, respectively, the phase portraits in the $v_{x}-v_{\varphi}$ plane are captured, as shown in Figures 13(c) and 13(d). Ignoring some tiny differences between numerical simulations and breadboard experiments due to the computational errors and parasitic circuit parameters, the experimental results are almost the same as the numerical simulations, which imply that the coexisting asymmetric attractors' behaviors that emerged from the memristive HR neuron model can be validated experimentally. 


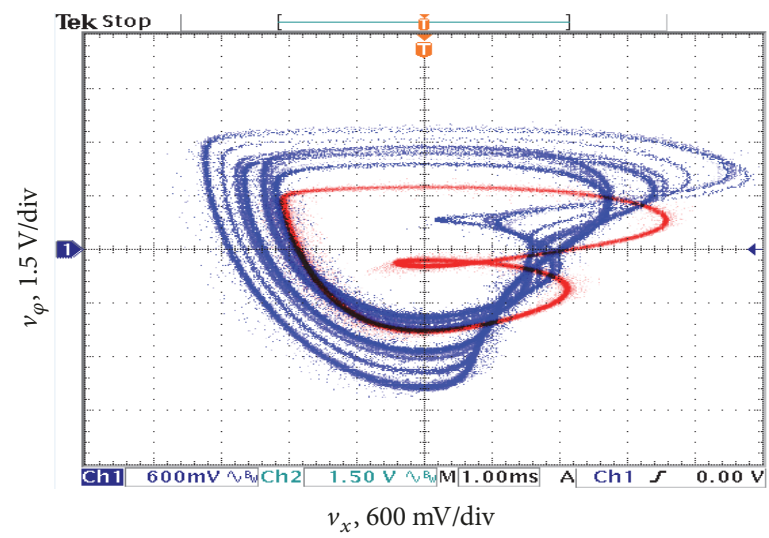

(a)

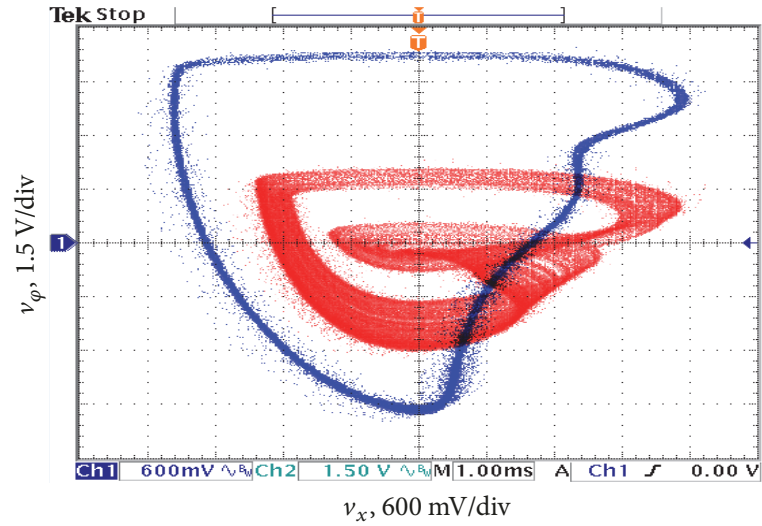

(c)

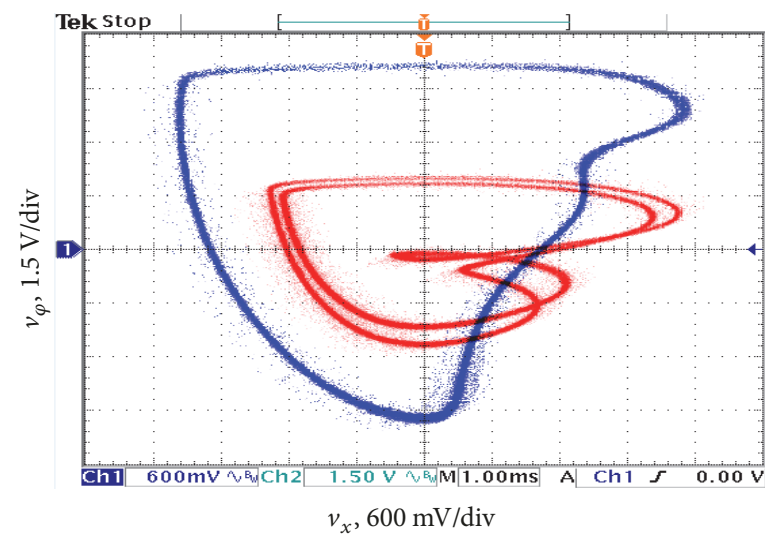

(b)

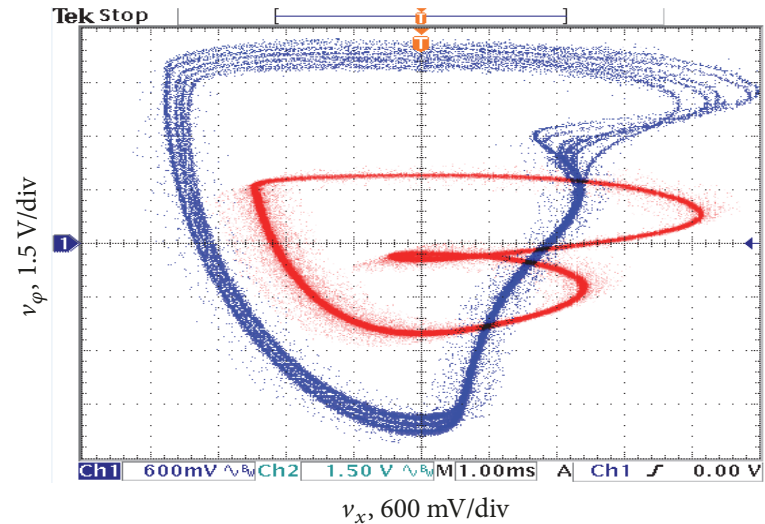

(d)

FIGURE 13: Experimentally measured phase portraits in the $v_{x}-v_{\varphi}$ plane for hidden coexisting asymmetric attractors under different circuit parameters. (a) $R_{1}=10 \mathrm{k} \Omega$ and $R_{k}=1.36 \mathrm{k} \Omega$. (b) $R_{1}=10 \mathrm{k} \Omega$ and $R_{k}=1.23 \mathrm{k} \Omega$. (c) $R_{1}=8.70 \mathrm{k} \Omega$ and $R_{k}=1.11 \mathrm{k} \Omega$. (d) $R_{1}=6.17 \mathrm{k} \Omega$ and $R_{k}=$ $1.11 \mathrm{k} \Omega$.

\section{Conclusions}

This paper presents a novel three-dimensional memristive HR neuron model to describe complex dynamics of neuronal activities with electromagnetic induction. The most prominent feature of this neuron model is that it does not contain any equilibrium point but can exhibit hidden coexisting behaviors of asymmetric attractors. Through executing bifurcation analyses, phase portraits, attraction basins, and dynamical maps, hidden coexisting asymmetric attractors are uncovered from the mathematical model and verified from the corresponding breadboard experiments. Thus, the proposed memristive HR neuron model can imitate the complex dynamical behaviors of electrical activities in neuron with electromagnetic induction. Further investigations will be performed in our future works.

\section{Conflicts of Interest}

The authors declare that they have no conflicts of interest.

\section{Acknowledgments}

This work was supported by the grants from the National Natural Science Foundations of China under Grant nos.
51777016, 51607013, 11602035, and 61601062 and the Natural Science Foundations of Jiangsu Province, China, under Grant no. BK20160282.

\section{References}

[1] A. L. Hodgkin and A. F. Huxley, "A quantitative description of membrane current and its application to conduction and excitation in nerve.," The Journal of Physiology, vol. 117, no. 4, pp. 500-544, 1952.

[2] J. L. Hindmarsh and R. M. Rose, "A model of the nerve impulse using two first-order differential equations," Nature, vol. 296, no. 5853, pp. $162-164,1982$.

[3] J. L. Hindmarsh and R. M. Rose, "A model of neuronal bursting using three coupled first order differential equations," Proceedings of the Royal Society of London B: Biological Sciences, vol. 221, no. 1222, pp. 87-102, 1984.

[4] C. R. Laing and C. C. Chow, "A spiking neuron model for binocular rivalry," Journal of Computational Neuroscience, vol. 12, no. 1, pp. 39-53, 2002.

[5] K. Tsumoto, H. Kitajima, T. Yoshinaga, K. Aihara, and H. Kawakami, "Bifurcations in Morris-Lecar neuron model," Neurocomputing, vol. 69, no. 4-6, pp. 293-316, 2006.

[6] H. Gu and B. Pan, "A four-dimensional neuronal model to describe the complex nonlinear dynamics observed in the firing 
patterns of a sciatic nerve chronic constriction injury model," Nonlinear Dynamics, vol. 81, no. 4, pp. 2107-2126, 2015.

[7] F. Wu, C. Wang, and Y. Xu, "Model of electrical activity in cardiac tissue under electromagnetic induction," Scientific Reports, vol. 6, article 28, 2016.

[8] J. Ma and J. Tang, "A review for dynamics in neuron and neuronal network," Nonlinear Dynamics, vol. 89, no. 3, pp. 15691578, 2017.

[9] M. Ge, Y. Jia, Y. Xu, and L. Yang, "Mode transition in electrical activities of neuron driven by high and low frequency stimulus in the presence of electromagnetic induction and radiation," Nonlinear Dynamics, vol. 91, no. 1, pp. 515-523, 2018.

[10] M. Storace, D. Linaro, and E. de Lange, "The HindmarshRose neuron model: bifurcation analysis and piecewise-linear approximations," Chaos: An Interdisciplinary Journal of Nonlinear Science, vol. 18, no. 3, Article ID 033128, 2008.

[11] G. Innocenti, A. Morelli, R. Genesio, and A. Torcini, "Dynamical phases of the Hindmarsh-Rose neuronal model: studies of the transition from bursting to spiking chaos," Chaos: An Interdisciplinary Journal of Nonlinear Science, vol. 17, no. 4, Article ID 043128, 2007.

[12] J. M. González-Miranda, "Complex bifurcation structures in the Hindmarsh-Rose neuron model," International Journal of Bifurcation and Chaos, vol. 17, no. 9, pp. 3071-3083, 2007.

[13] J. M. González-Miranda, "Observation of a continuous interior crisis in the Hindmarsh-Rose neuron model," Chaos: An Interdisciplinary Journal of Nonlinear Science, vol. 13, no. 3, pp. 845852, 2003.

[14] G. Innocenti and R. Genesio, "On the dynamics of chaotic spiking-bursting transition in the Hindmarsh-Rose neuron," Chaos: An Interdisciplinary Journal of Nonlinear Science, vol. 19, no. 2, Article ID 023124, 2009.

[15] T. Wei, B. Ou, J. Li et al., "Transcriptional profiling of rice early response to Magnaporthe oryzae identified OsWRKYs as important regulators in rice blast resistance," PLOS ONE, vol. 8, no. 3, Article ID e59720, 2013.

[16] S. R. D. Djeundam, R. Yamapi, T. C. Kofane, and M. A. Azizalaoui, "Deterministic and stochastic bifurcations in the Hindmarsh-Rose neuronal model," Chaos: An Interdisciplinary Journal of Nonlinear Science, vol. 23, no. 3, Article ID 033125, 2013.

[17] E. B. Ngouonkadi, H. B. Fotsin, P. Louodop Fotso, V. Kamdoum Tamba, and H. A. Cerdeira, "Bifurcations and multistability in the extended Hindmarsh-Rose neuronal oscillator," Chaos, Solitons \& Fractals, vol. 85, pp. 151-163, 2016.

[18] K. Wu, T. Luo, H. Lu, and Y. Wang, "Bifurcation study of neuron firing activity of the modified Hindmarsh-Rose model," Neural Computing and Applications, vol. 27, no. 3, pp. 739-747, 2016.

[19] S. K. Thottil and R. P. Ignatius, "Nonlinear feedback coupling in Hindmarsh-Rose neurons," Nonlinear Dynamics, vol. 87, no. 3 , pp. 1879-1899, 2017.

[20] H. Wang, Y. Zheng, and Q. Lu, "Stability and bifurcation analysis in the coupled HR neurons with delayed synaptic connection," Nonlinear Dynamics, vol. 88, no. 3, pp. 2091-2100, 2017.

[21] S. Lakshmanan, C. P. Lim, S. Nahavandi, M. Prakash, and P. Balasubramaniam, "Dynamical analysis of the HindmarshRose neuron with time delays," IEEE Transactions on Neural Networks and Learning Systems, vol. 28, no. 8, pp. 1953-1958, 2017.
[22] H. Wang, Q. Wang, and Y. Zheng, "Bifurcation analysis for Hindmarsh-Rose neuronal model with time-delayed feedback control and application to chaos control," Science China Technological Sciences, vol. 57, no. 5, pp. 872-878, 2014.

[23] D. Jun, Z. Guang-Jun, X. Yong, Y. Hong, and W. Jue, "Dynamic behavior analysis of fractional-order Hindmarsh-Rose neuronal model," Cognitive Neurodynamics, vol. 8, no. 2, pp. 167175, 2014.

[24] E. Kaslik, "Analysis of two- and three-dimensional fractionalorder Hindmarsh-Rose type neuronal models," Fractional Calculus and Applied Analysis, vol. 20, no. 3, pp. 623-645, 2017.

[25] M. Lv, C. Wang, G. Ren, J. Ma, and X. Song, "Model of electrical activity in a neuron under magnetic flow effect," Nonlinear Dynamics, vol. 85, no. 3, pp. 1479-1490, 2016.

[26] M. Lv and J. Ma, "Multiple modes of electrical activities in a new neuron model under electromagnetic radiation," Neurocomputing, vol. 205, pp. 375-381, 2016.

[27] L. Lu, Y. Jia, W. Liu, and L. Yang, "Mixed stimulus-induced mode selection in neural activity driven by high and low frequency current under electromagnetic radiation," Complexity, vol. 2017, Article ID 7628537, pp. 1-11, 2017.

[28] S. Jafari, J. C. Sprott, and S. M. R. H. Golpayegani, "Elementary quadratic chaotic flows with no equilibria," Physics Letters A, vol. 377, no. 9, pp. 699-702, 2013.

[29] V.-T. Pham, C. Volos, S. Jafari, Z. Wei, and X. Wang, "Constructing a novel no-equilibrium chaotic system," International Journal of Bifurcation and Chaos, vol. 24, no. 5, Article ID 1450073, 2014.

[30] F. R. Tahir, S. Jafari, V.-T. Pham, C. Volos, and X. Wang, "A novel no-equilibrium chaotic system with multiwing butterfly attractors," International Journal of Bifurcation and Chaos, vol. 25, no. 4, Article ID 1550056, 2015.

[31] A. P. Kuznetsov, S. P. Kuznetsov, E. Mosekilde, and N. V. Stankevich, "Co-existing hidden attractors in a radio-physical oscillator system," Journal of Physics A: Mathematical and Theoretical, vol. 48, no. 12, Article ID 125101, 2015.

[32] B. Bao, H. Qian, Q. Xu, M. Chen, J. Wang, and Y. Yu, "Coexisting behaviors of asymmetric attractors in hyperbolictype memristor based hopfield neural network," Frontiers in Computational Neuroscience, vol. 11, article 81, 2017.

[33] A. Shilnikov, R. L. Calabrese, and G. Cymbalyuk, "Mechanism of bistability: tonic spiking and bursting in a neuron model," Physical Review E: Statistical, Nonlinear, and Soft Matter Physics, vol. 71, no. 2, Article ID 056214, 2005.

[34] B. Bao, H. Qian, J. Wang et al., "Numerical analyses and experimental validations of coexisting multiple attractors in Hopfield neural network," Nonlinear Dynamics, vol. 90, no. 4, pp. 2359-2369, 2017.

[35] T. Malashchenko, A. Shilnikov, and G. Cymbalyuk, "Six types of multistability in a Neuronal model based on slow calcium current," PLoS ONE, vol. 6, no. 7, Article ID e21782, 2011.

[36] L. Chua, "Resistance switching memories are memristors," Applied Physics A: Materials Science \& Processing, vol. 102, no. 4, pp. 765-783, 2011.

[37] L. O. Chua, "The fourth element," Proceedings of the IEEE, vol. 100, no. 6, pp. 1920-1927, 2012.

[38] S. P. Adhikari, M. P. Sah, H. Kim, and L. O. Chua, "Three fingerprints of memristor," IEEE Transactions on Circuits and Systems II: Express Briefs, vol. 60, no. 11, pp. 3008-3021, 2013.

[39] A. Wolf, J. B. Swift, and H. L. a. Swinney, "Determining Lyapunov exponents from a time series," Physica D: Nonlinear Phenomena, vol. 16, no. 3, pp. 285-317, 1985. 
[40] P. C. Rech, "Period-adding and spiral organization of the periodicity in a Hopfield neural network," International Journal of Machine Learning and Cybernetics, vol. 6, no. 1, 2013.

[41] H. Bao, N. Wang, B. Bao, M. Chen, P. Jin, and G. Wang, "Initial condition-dependent dynamics and transient period in memristor-based hypogenetic jerk system with four line equilibria," Communications in Nonlinear Science and Numerical Simulation, vol. 57, pp. 264-275, 2018.

[42] B. C. Bao, H. Bao, N. Wang, M. Chen, and Q. Xu, "Hidden extreme multistability in memristive hyperchaotic system," Chaos, Solitons \& Fractals, vol. 94, pp. 102-111, 2017.

[43] K. Rajagopal, A. Akgul, S. Jafari, and B. Aricioglu, "A chaotic memcapacitor oscillator with two unstable equilibriums and its fractional form with engineering applications," Nonlinear Dynamics, vol. 91, no. 2, pp. 957-974, 2018.

[44] K. Rajagopal, L. Guessas, A. Karthikeyan, A. Srinivasan, and G. Adam, "Fractional order memristor no equilibrium chaotic system with its adaptive sliding mode synchronization and genetically optimized fractional order PID synchronization," Complexity, vol. 2017, Article ID 1892618, pp. 1-19, 2017.

[45] K. Rajagopal, A. Karthikeyan, and A. K. Srinivasan, "FPGA implementation of novel fractional-order chaotic systems with two equilibriums and no equilibrium and its adaptive sliding mode synchronization," Nonlinear Dynamics, vol. 87, no. 4, pp. 2281-2304, 2017.

[46] B. Bao, T. Jiang, G. Wang, P. Jin, H. Bao, and M. Chen, “Twomemristor-based Chua's hyperchaotic circuit with plane equilibrium and its extreme multistability," Nonlinear Dynamics, vol. 89, no. 2, pp. 1157-1171, 2017. 


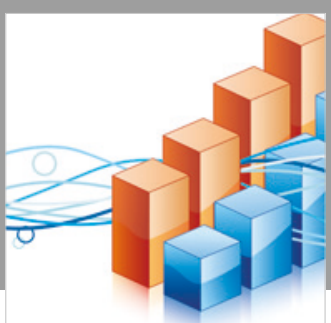

Advances in

Operations Research

\section{-n-m}
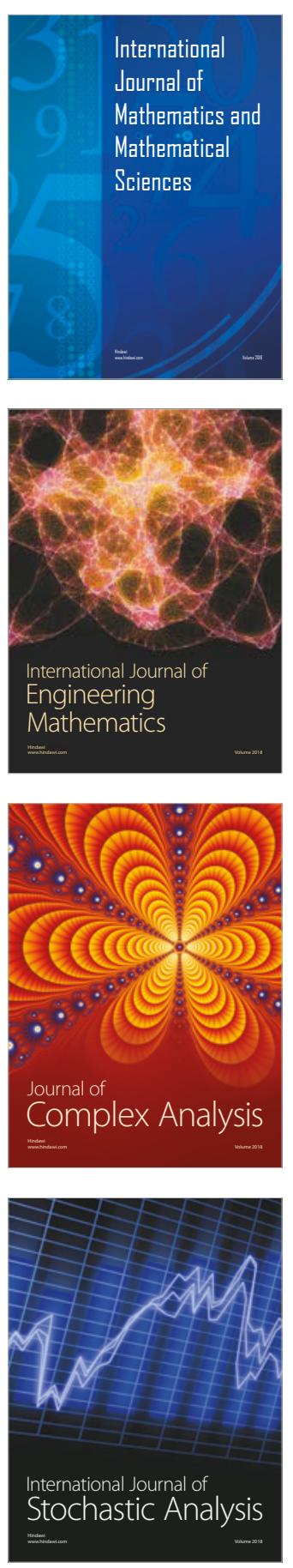
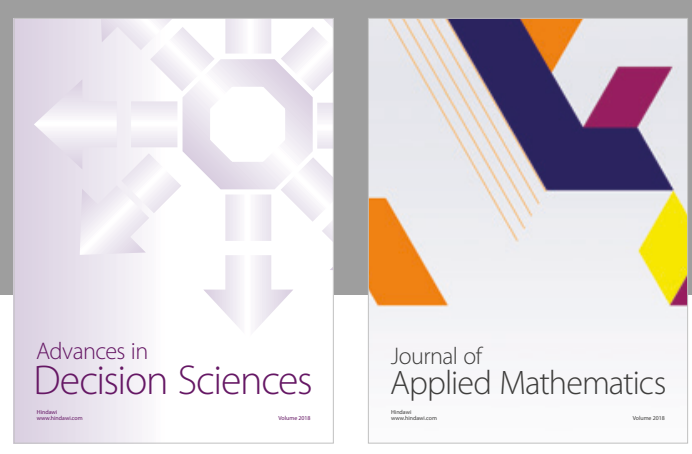

Journal of

Applied Mathematics
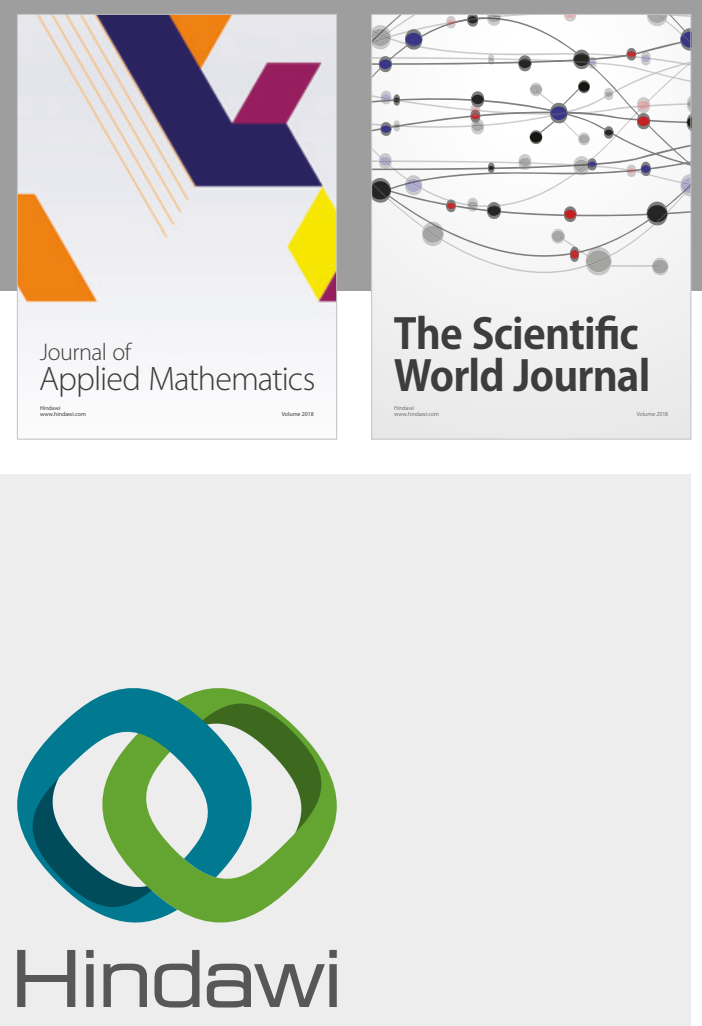

Submit your manuscripts at

www.hindawi.com

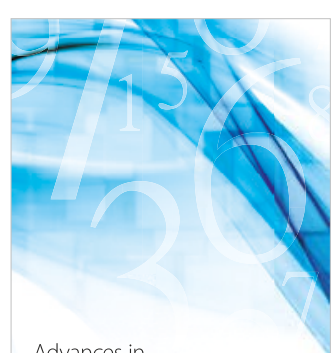

Advances in
Numerical Analysis
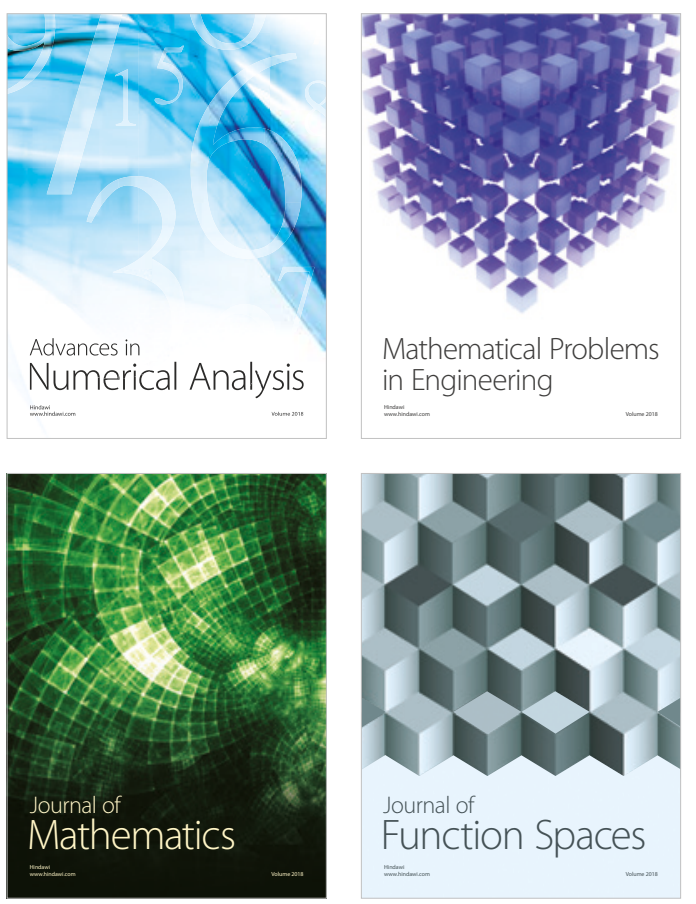

Mathematical Problems in Engineering

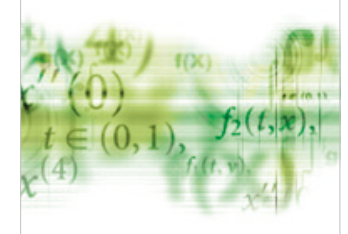

International Journal of

Differential Equations

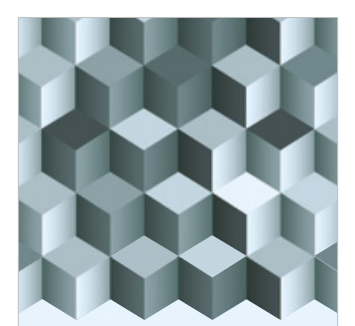

Journal of

Function Spaces
The Scientific

World Journal

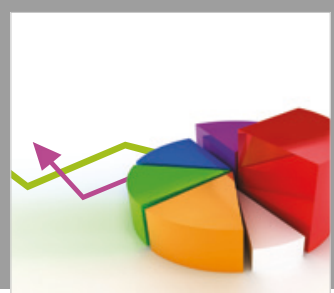

Journal of

Probability and Statistics
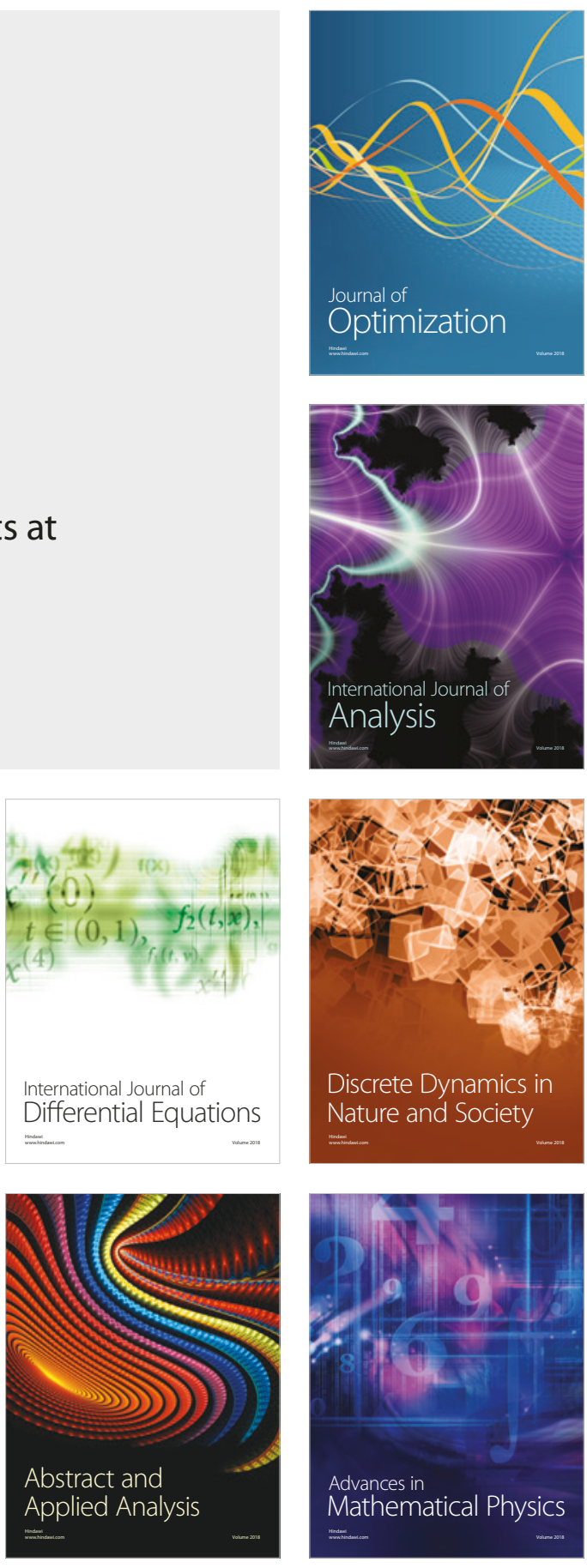